\title{
INFLUÊNCIA DA ÉPOCA DE SEMEADURA NO COMPORTAMENTO DE CULTIVARES DE ARROZ IRRIGADO POR ASPERSÃO EM SELVÍRIA, MS'
}

\author{
ORIVALDO ARF², RICARDO ANTÔNIO FERREIRA RODRIGUES ${ }^{3}$, \\ MARCO EUSTÁQUIO DE SÁ ${ }^{2}$ e CARLOS ALEXANDRE COSTA CRUSCIOL ${ }^{4}$
}

\begin{abstract}
RESUMO - Este trabalho teve por objetivo avaliar os efeitos da época da semeadura no comportamento de diferentes cultivares de arroz (Oryza sativa L.) de sequeiro (IAC 201, Carajás, Guarani, IAC 202, CNA 7800, CNA 7801, Caiapó, Rio Paranaíba e Araguaia) irrigados por aspersão, quanto à produção e qualidade dos grãos. As sementes foram semeadas no início da segunda quinzena dos meses de setembro, outubro, novembro, dezembro, janeiro e fevereiro. Os experimentos foram instalados no Município de Selvíria, MS, durante os anos agrícolas 1995/96 e 1996/97. O delineamento experimental utilizado foi o de blocos casualizados, com quatro repetições. O controle da irrigação foi realizado por meio de tensiômetros, e a suplementação hídrica foi realizada quando o potencial matricial atingiu $-0,033 \mathrm{MPa}$, durante a fase reprodutiva e -0,058 MPa, nas demais fases. De acordo com os resultados obtidos, pode-se concluir que as cultivares CNA 7801, Carajás, IAC 201, CNA 7800 e IAC 202 apresentaram comportamento superior; a semeadura realizada em novembro propiciou produtividade mais elevada; semeaduras antecipadas (setembro-outubro) ou retardada (fevereiro) resultaram em menores índices de acamamento; as cultivares IAC 202, CNA 7800 e CNA 7801 apresentaram ausência ou baixo índice de acamamento, nas diferentes épocas de semeadura e, a semeadura retardada (fevereiro) proporcionou a obtenção de maior rendimento de inteiros.
\end{abstract}

Termos para indexação: Oryza sativa, grãos, componentes de rendimento, produtividade, qualidade, acamamento, arroz de sequeiro.

\section{EFFECTS OF SOWING DATES ON PERFORMANCE OF DRYLAND RICE CULTIVARS UNDER SPRINKLER IRRIGATION, IN SELVÍRIA, MS, BRAZIL}

\begin{abstract}
This study was carried out to evaluate the effects of sowing periods on the performance of dryland rice (Oryza sativa L.) (IAC 201, Carajás, Guarani, IAC 202, CNA 7800, CNA 7801, Caiapó, Rio Paranaíba and Araguaia cultivars) under sprinkler irrigation. The seeds were sowed at the beginning of the second fortnight of September, October, November, December, January and February. The experiments were conducted in Selvíria, MS, Brazil, during the agricultural years of 1995/96 and 1996/97. The experimental design used in each sowing was in randomized blocks, with four replications. The irrigation control was done through tensiometer and the water supply was established when the matric potential reached $-0.033 \mathrm{MPa}$, during the reproductive phase and $-0.058 \mathrm{MPa}$, in the other phases. The results showed that CNA 7801, Carajás, IAC 201, CNA 7800, and IAC 202 presented better performance; November sowing reached higher productivity; earlier sowings (September-October) or delayed (February) caused smaller laying indexes; IAC 202, CNA 7800, and CNA 7801 presented absence or lower laying index, in the different sowing times and, in delayed sowing (February) larger whole grain rate was obtained.
\end{abstract}

Index terms: Oryza sativa, grain, yield components, productivity, quality, layering, upland rice.

\footnotetext{
${ }^{1}$ Aceito para publicação em 28 de dezembro de 1999. Trabalho desenvolvido com apoio financeiro da FAPESP e do CNPq.

${ }^{2}$ Eng. Agrôn., Dr., Dep. de Fitotecnia, Economia e Sociologia Rural, Faculdade de Engenharia (FE), Universidade Estadual Paulista (UNESP), Caixa Postal 31, CEP 15385-000
}

Ilha Solteira, SP. E-mail: gd@adm.feis.unesp.br, mesa@agr.feis.unesp.br

${ }^{3}$ Eng. Agrôn., Dr., Dep. de Ciência do Solo e Engenharia Rural, FE, UNESP. E-mail: ricardo@ agr.feis.unesp.br

${ }^{4}$ Eng. Agrôn., Dr., Dep. de Agricultura e Melhoramento Vegetal, Faculdade de Ciências Agronômicas (FCA), UNESP, Caixa Postal 237, CEP 18603-970 Botucatu, SP. E-mail: secdamv@fca.unesp.br 


\section{INTRODUÇÃO}

No Brasil, cerca de $60 \%$ da produção de arroz (Oryza sativa L.) origina-se de lavouras de sequeiro, em que a água disponível para a planta provém somente das chuvas. As plantas cultivadas nesse sistema estão sujeitas a períodos de estiagens de duas a três semanas, que caracterizam os chamados veranicos. A deficiência hídrica, a alta demanda evapotranspirativa durante esse período e a baixa tecnologia normalmente utilizada reduzem a produtividade e podem até causar a perda total da lavoura. Uma das alternativas para a solução do problema é o uso da irrigação por aspersão, que, além de minimizar o risco de perda da lavoura, pode aumentar a produtividade e melhorar a qualidade dos grãos produzidos. Com a garantia conferida pelo uso da irrigação, o agricultor sente-se estimulado a usar um maior nível de tecnologia. Entretanto, a maioria das cultivares atualmente recomendadas para cultivo irrigado são do tipo tradicional, com forte tendência ao acamamento, quando cultivados em solo fértil ou com suprimento adequado de água e nutrientes.

O tipo de planta ideal para o cultivo irrigado por aspersão seria intermediário entre o tradicional de arroz de sequeiro e o moderno de arroz irrigado por aspersão (Pinheiro et al., 1985). Deve possuir basicamente as seguintes características: alta capacidade produtiva, resistência ao acamamento, ciclo precoce a médio, resistência à brusone e à mancha-parda, certo grau de dormência, grãos largos, finos e translúcidos (Sant'Ana, 1989).

A luz e a temperatura são os fatores climáticos que mais influenciam o comportamento da cultura do arroz (Chang \& Oka, 1976; Vergara, 1976; Chaudhary \& Sodhi, 1979; Yoshida, 1981). Poucas são as informações sobre épocas de semeadura do arroz de sequeiro irrigado por aspersão; o que se sabe é que para o arroz irrigado, o período de semeadura, na região sul do Brasil, é curto, em comparação com o de regiões com menores latitudes. Assim, no Rio Grande do Sul, a época de semeadura mais adequada é outubro-novembro (Amaral, 1979; Infeld, 1984, 1987). Outubro-novembro também é o período mais indicado para a semeadura no sul de Minas Gerais (Morais et al., 1978) e em São Paulo (Fornasieri Filho \& Fornasieri, 1993). Em estados brasileiros sob menor latitude, o período de semeadura é mais longo. Em Goiás, a semeadura antecipada, em agosto, propiciou obtenção de maior produção (Santos et al., 1978). De acordo com Sant'Ana (1989), a semeadura do arroz de sequeiro irrigado por aspersão deve ser realizada na época normal de semeadura do arroz de sequeiro.

Embora o sistema de cultivo do arroz irrigado por aspersão seja prática recente, a área de cultivo está se expandindo rapidamente, principalmente no Brasil central, carecendo, porém, de informações técnicas para sua perfeita implementação (Sant'Ana, 1989). Este trabalho teve como objetivo avaliar os efeitos da época de semeadura no comportamento de diversas cultivares de arroz de sequeiro, irrigadas por aspersão, quanto à produção e qualidade de grãos.

\section{MATERIAL E MÉTODOS}

Os experimentos foram conduzidos no município de Selvíria, MS, em área experimental pertencente a Faculdade de Engenharia de Ilha Solteira - UNESP, situada aproximadamente a $51^{\circ} 22^{\prime}$ de longitude Oeste de Greenwich e $20^{\circ} 22^{\prime}$ de latitude Sul, com altitude de 335 metros. Caracteriza-se, climaticamente, por uma temperatura média anual de $23,5^{\circ} \mathrm{C}$, umidade relativa do ar média anual entre $70 \%$ e $80 \%$ e a precipitação média anual de $1.370 \mathrm{~mm}$

O solo do local é do tipo Latossolo Vermelho-Escuro, epieutrófico álico, textura argilosa, e cultivado anteriormente com a cultura do milho. As características químicas foram determinadas antes da instalação dos experimentos, segundo a metodologia de Raij \& Quaggio (1983) e apresentou os seguintes resultados: M.O. $=30 \mathrm{~g} / \mathrm{dm}^{3}$; $\mathrm{P}($ resina $)=8,0 \mathrm{mg} / \mathrm{dm}^{3} ; \mathrm{pH}\left(\mathrm{CaCl}_{2}\right)=4,7 ; \mathrm{K}, \mathrm{Ca}, \mathrm{Mg}$, $\mathrm{H}+\mathrm{Al}$ e $\mathrm{Al}=2,9 ; 22,0 ; 4,0 ; 42,0$ e $5,0 \mathrm{mmol}_{\mathrm{c}} / \mathrm{dm}^{3}$, respectivamente $\mathrm{e}=41 \%$.

As chuvas que ocorreram durante a condução dos experimentos foram quantificadas diariamente através de pluviômetro instalado na área experimental.

O solo foi preparado por uma aração e duas gradagens, sendo a última realizada às vésperas da semeadura. A seguir, realizou-se a sulcação mecânica, no espaçamento de $0,40 \mathrm{~m}$ entre fileiras.

A adubação química básica nos sulcos de semeadura constou da aplicação de $250 \mathrm{~kg} / \mathrm{ha}$ da formulação 4-30-10 + 0,4\% Zn. Na adubação de cobertura foram utilizados $30 \mathrm{~kg} / \mathrm{ha}$ de $\mathrm{N}$ na forma de sulfato de amônio, ao redor de 35 dias após a emergência das plantas. 
A semeadura foi realizada manualmente, no início da segunda quinzena de cada mês: 19/setembro, 20/outubro, 17/novembro, 19/dezembro, 18/janeiro e 16/fevereiro, no ano agrícola de 1995/96 e 19/setembro, 18/outubro, 18/novembro, 18/dezembro, 20/janeiro e 18/fevereiro de 1996/97, utilizando-se número de sementes necessário para se obter um estande em torno de 120 plantas $/ \mathrm{m}^{2}$. Junto com as sementes aplicou-se 1,5 kg/ha de carbofuran (i.a.), visando principalmente ao controle de cupins e lagartaselasmo.

O delineamento experimental utilizado foi o de blocos ao acaso, com quatro repetições. As parcelas foram constituídas por seis linhas de 4,5 m de comprimento, totalizando uma área de $10,8 \mathrm{~m}^{2}$, sendo a área útil constituída pelas quatro linhas centrais, desprezando-se $0,5 \mathrm{~m}$ em ambas as extremidades de cada linha.

As cultivares utilizadas foram as seguintes: IAC 201, Carajás, Guarani, IAC 202, CNA 7800, CNA 7801, Caiapó, Rio Paranaíba e Araguaia.

As irrigações foram realizadas por um sistema convencional de aspersão; e para determinar a necessidade de irrigação, utilizaram-se tensiômetros, colocados a $10-20 \mathrm{~cm}$ de profundidade, conforme método proposto por Faria (1987).

O controle de plantas daninhas foi realizado com aplicação do herbicida pendimenthalin, na dose de $1.100 \mathrm{~g} / \mathrm{ha}$ do ingrediente ativo. As plantas daninhas não atingidas pelo herbicida foram eliminadas por meio de capina manual.

Quando 90\% das panículas apresentavam os grãos com coloração típica de maduros, realizou-se a colheita manualmente utilizando-se altura de corte de 15 a $20 \mathrm{~cm}$. Em seguida, foi realizada a secagem ao sol, durante um a dois dias, em terreiro cimentado, e, posteriormente, a trilha mecânica.

Foram avaliadas as seguintes características: floração e maturação, ou seja, o número de dias transcorridos entre a emergência e a floração de $50 \%$ das plantas e entre a emergência e a maturação de $90 \%$ das panículas das parcelas; altura das plantas, pela determinação, durante o estádio de grãos na forma pastosa, em dez plantas ao acaso, na área útil de cada parcela, da distância média compreendida desde a superfície do solo até a extremidade da panícula mais alta esticada para cima; acamamento: obtido por meio de observações visuais na fase de maturação, utilizando-se a seguinte escala de notas: 0 - sem acamamento; 1 - até 5\% de plantas acamadas; 2 - 5\% a $25 \% ; 3-25 \%$ a $50 \% ; 4-50 \%$ a $75 \%$ e $5-75 \%$ a $100 \%$ de plantas acamadas; número de panículas $/ \mathrm{m}^{2}$ : determinado mediante a contagem das panículas coletadas, e, posteriormente, realizada matematicamente a conversão para número de panículas $/ \mathrm{m}^{2}$; produção de grãos, obtida por meio de pesagem dos grãos em casca, proveniente da área útil das parcelas, corrigindo-se a umidade para $13 \%$ e convertendo-a em kg/ha; e rendimento de inteiros (retirou-se uma amostra de $100 \mathrm{~g}$ de arroz em casca passando-a em engenho de prova Suzuki, modelo MT, por 1 minuto; em seguida foram pesados os grãos brunidos assim obtidos, e o valor encontrado foi considerado como rendimento de benefício, em porcentagem, posteriormente, os grãos brunidos foram colocados no "trieur" no 2 , e a separação dos grãos foi processada por 0,5 minuto; foram pesados os grãos que permaneceram no "trieur", e o valor encontrado foi considerado rendimento de inteiros).

\section{RESULTADOS E DISCUSSÃO}

Os valores médios da umidade relativa do ar (\%), precipitação (mm) e temperatura máxima e mínima $\left({ }^{\circ} \mathrm{C}\right)$ observados durante a condução dos experimentos nos dois anos agrícolas (1995/96 e 1996/97), estão apresentados nas Figs. 1 e 2. Pelos dados apresentados, pode-se observar que o ano agrícola 1996/97 apresentou maiores valores de precipitação e umidade relativa no período de setembro a maio. No ano agrícola 1995/96, houve maior desuniformidade de distribuição de chuvas, pois ocorreu baixa precipitação em novembro/1995. No que se refere à temperatura, os maiores valores ocorreram em dezembro, janeiro e fevereiro, e a partir de março a temperatura começou a declinar; entretanto, mesmo no mês de maio a temperatura mínima média foi superior a $15^{\circ} \mathrm{C}$. No primeiro ano agrícola, a temperatura mínima diária foi inferior a $15^{\circ} \mathrm{C}$ somente nos dias 18, 19 e 20 de abril, e 20 e 31 de maio de 1996. Já no segundo ano agrícola, valores inferiores a $15^{\circ} \mathrm{C}$ foram observados em 27 de abril, 29 e 31 de maio de 1997.

Os resultados obtidos na avaliação do número de dias para florescimento e ciclo das cultivares de arroz utilizadas nas diferentes épocas de semeadura estão apresentados na Tabela 1. Pelos dados, verificouse que as cultivares IAC 201, Carajás e Guarani apresentaram, em cada época de semeadura, número de dias para florescimento muito próximos e o mesmo ciclo nos dois anos de cultivo. Comportamento semelhante ocorreu com as cultivares Caiapó, Rio Paranaíba e Araguaia. Os resultados obtidos são concordantes com Arf (1993), Oliveira (1994) e Nakao 
(1995). Quanto ao ciclo, verificou-se, ainda, que nos dois anos de cultivo, as cultivares IAC 201, Carajás e Guarani apresentaram ciclo mais curto, ou seja: 80 a 104 dias no ano agrícola 1995/96 e de 90 a 96 dias no ano agrícola 1996/97. Já as cultivares Caiapó, Rio Paranaíba e Araguaia apresentaram ciclo mais

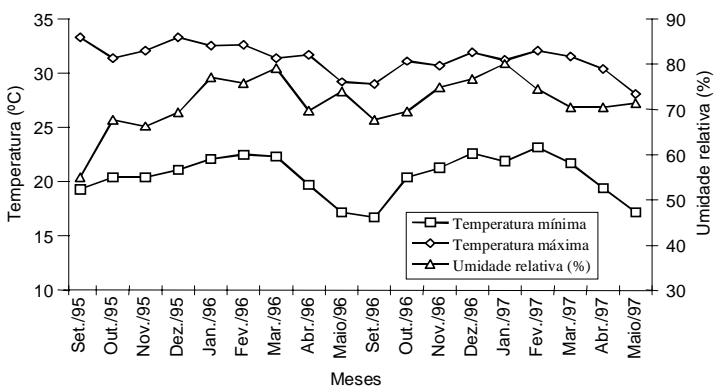

FIG. 1. Variação de temperatura e umidade relativa durante a condução dos experimentos. Selvíria, MS. longo, de 97 a 116 dias (1995/96) e 103 a 113 dias (1996/97), enquanto as cultivares IAC 202, CNA 7800 e CNA 7801 apresentaram valores inter-

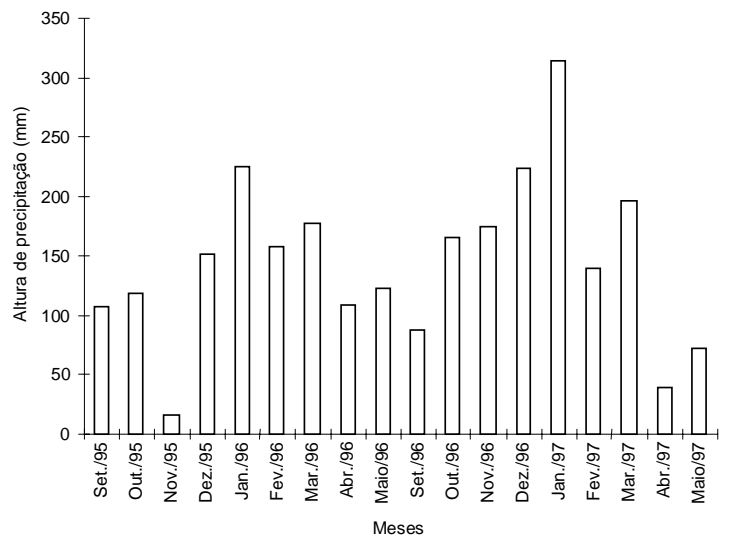

FIG. 2. Ocorrência de precipitação pluvial durante a condução dos experimentos. Selvíria, MS.

TABELA 1. Número de dias da emergência ao florescimento (f) e da emergência à colheita (c), de cultivares de arroz semeadas em diferentes épocas em cultivo irrigado por aspersão. Selvíria, MS, 1995/96 e $1996 / 97$.

\begin{tabular}{|c|c|c|c|c|c|c|c|c|c|c|c|c|c|c|}
\hline \multirow[t]{3}{*}{ Cultivar } & \multicolumn{12}{|c|}{ Época de semeadura } & \multicolumn{2}{|c|}{ Média } \\
\hline & \multicolumn{2}{|c|}{ Set. } & \multicolumn{2}{|c|}{ Out. } & \multicolumn{2}{|c|}{ Nov. } & \multicolumn{2}{|c|}{ Dez. } & \multicolumn{2}{|c|}{ Jan. } & \multicolumn{2}{|c|}{ Fev. } & \multirow[t]{2}{*}{$\mathrm{f}$} & \multirow[t]{2}{*}{$\mathrm{c}$} \\
\hline & $\mathrm{f}$ & $\mathrm{c}$ & $\mathrm{f}$ & $\mathrm{c}$ & $\mathrm{f}$ & $\mathrm{c}$ & $f$ & $\mathrm{c}$ & $\mathrm{f}$ & $\mathrm{c}$ & $\mathrm{f}$ & $\mathrm{c}$ & & \\
\hline & \multicolumn{14}{|c|}{$1995 / 96$} \\
\hline IAC 201 & 80 & 104 & 70 & 96 & 65 & 97 & 58 & 84 & 57 & 80 & 54 & 82 & 64 & 90 \\
\hline Carajás & 79 & 104 & 71 & 96 & 67 & 97 & 59 & 84 & 58 & 80 & 53 & 82 & 64 & 90 \\
\hline Guarani & 77 & 104 & 70 & 96 & 65 & 97 & 59 & 84 & 58 & 80 & 53 & 82 & 64 & 90 \\
\hline IAC 202 & 93 & 110 & 87 & 108 & 76 & 106 & 72 & 95 & 68 & 88 & 69 & 99 & 77 & 101 \\
\hline CNA 7800 & 86 & 107 & 74 & 104 & 72 & 103 & 63 & 90 & 62 & 84 & 61 & 95 & 70 & 97 \\
\hline CNA 7801 & 90 & 107 & 79 & 103 & 73 & 103 & 67 & 90 & 63 & 84 & 63 & 95 & 72 & 97 \\
\hline Caiapó & 93 & 111 & 86 & 112 & 80 & 110 & 76 & 98 & 75 & 97 & 81 & 116 & 82 & 107 \\
\hline R. Paranaíba & 93 & 112 & 87 & 112 & 80 & 110 & 76 & 98 & 75 & 97 & 81 & 116 & 82 & 107 \\
\hline Araguaia & 93 & 111 & 89 & 112 & 82 & 110 & 77 & 98 & 76 & 97 & 81 & 116 & 83 & 107 \\
\hline \multirow[t]{2}{*}{ Média } & 87 & 108 & 79 & 104 & 73 & 104 & 67 & 91 & 65 & 87 & 66 & 98 & & \\
\hline & \multicolumn{14}{|c|}{$1996 / 97$} \\
\hline IAC 201 & 70 & 96 & 65 & 95 & 65 & 95 & 64 & 90 & 59 & 90 & 62 & 91 & 64 & 93 \\
\hline Carajás & 70 & 96 & 65 & 95 & 66 & 95 & 64 & 90 & 59 & 90 & 62 & 91 & 64 & 93 \\
\hline Guarani & 70 & 96 & 64 & 95 & 66 & 95 & 64 & 90 & 58 & 90 & 62 & 91 & 64 & 93 \\
\hline IAC 202 & 80 & 106 & 78 & 108 & 78 & 107 & 77 & 105 & 71 & 101 & 73 & 101 & 76 & 105 \\
\hline CNA 7800 & 74 & 102 & 67 & 101 & 72 & 100 & 68 & 102 & 65 & 95 & 68 & 98 & 69 & 100 \\
\hline CNA 7801 & 75 & 102 & 71 & 103 & 75 & 101 & 69 & 102 & 65 & 95 & 70 & 100 & 71 & 101 \\
\hline Caiapó & 84 & 113 & 80 & 110 & 78 & 107 & 76 & 105 & 73 & 103 & 75 & 110 & 78 & 108 \\
\hline R. Paranaíba & 84 & 113 & 80 & 110 & 78 & 107 & 76 & 105 & 73 & 103 & 75 & 110 & 78 & 108 \\
\hline Araguaia & 84 & 113 & 79 & 110 & 78 & 107 & 76 & 105 & 73 & 103 & 75 & 110 & 78 & 108 \\
\hline Média & 77 & 104 & 72 & 103 & 73 & 102 & 70 & 99 & 66 & 97 & 69 & 100 & - & - \\
\hline
\end{tabular}


mediários. Pode-se ainda verificar que tanto o número de dias para florescimento, quanto o ciclo das cultivares, nos dois anos de cultivo, apresentaram uma diminuição, se for comparada a semeadura realizada em setembro, com a realizada em janeiro/fevereiro. Este comportamento pode ser explicado pela variação principalmente da temperatura entre as diferentes épocas de semeadura (Fig. 1). Na mesma figura, pode-se observar que os valores de temperatura diminuíram a partir do mês de fevereiro. Os resultados são concordantes com Chang \& Oka (1976), Vergara (1976), Chaudhary \& Sodhi (1979) e Yoshida (1981).

Os resultados obtidos na avaliação da altura de plantas, grau de acamamento e número de panículas $/ \mathrm{m}^{2}$, produção de grãos e rendimento de inteiros estão apresentados nas Tabelas 2 a 5 e Figs. 3 e 4 . Houve efeito significativo no que diz respeito a cultivares, época de semeadura, e da interação cultivar x época de semeadura referente a todas as características avaliadas nos dois anos de cultivo, com exceção da interação cultivar x época de semeadura no ano agrícola 1996/97, referente ao número de panícula/m² . Pela Tabela 2, verifica-se que, de maneira geral, as cultivares CNA 7800, CNA 7801 e IAC 202 apresentaram os menores valores quanto à altura das plantas, em todas as épocas de semeadura, nos dois anos de cultivo. Já as cultivares Caiapó, Rio Paranaíba e Araguaia apresentaram os maiores valores de altura das plantas, e as cultivares IAC 201, Carajás e Guarani, valores intermediários. No que se refere à época de semeadura, quando realizada nos meses de novembro e dezembro de 1995/96 e novembro de 1996/97, verificou-se a obtenção dos maiores valores quanto à altura das plantas, o que pode ser explicado em razão da ocorrência de temperaturas mais

TABELA 2. Altura das plantas (cm) de nove cultivares de arroz em diferentes épocas de semeadura. Selvíria, MS, 1995/96 e 1996/971.

\begin{tabular}{|c|c|c|c|c|c|c|c|}
\hline \multirow[t]{2}{*}{ Cultivar } & \multicolumn{6}{|c|}{ Época de semeadura } & \multirow[t]{2}{*}{ Média } \\
\hline & Set. & Out. & Nov. & Dez. & Jan. & Fev. & \\
\hline & & & & $1995 / 96$ & & & \\
\hline IAC 201 & $96,50 \mathrm{cdD}$ & $111,50 \mathrm{cdBC}$ & $130,25 \mathrm{cdA}$ & $124,75 \mathrm{dA}$ & $121,50 \mathrm{bcAB}$ & $110,00 \mathrm{cC}$ & $115,75 \mathrm{c}$ \\
\hline Carajás & $103,50 \mathrm{bcC}$ & $116,75 \mathrm{bcB}$ & $137,25 \mathrm{bcA}$ & $139,25 \mathrm{bcA}$ & $130,00 \mathrm{abA}$ & $113,00 \mathrm{cBC}$ & $123,29 b$ \\
\hline Guarani & $103,00 \mathrm{bcC}$ & $119,00 \mathrm{bcB}$ & $134,00 \mathrm{cdA}$ & $138,75 \mathrm{cA}$ & $131,50 \mathrm{abA}$ & $115,75 \mathrm{bcB}$ & $123,67 b$ \\
\hline IAC 202 & $88,50 \mathrm{deD}$ & $100,75 \mathrm{deBC}$ & $123,50 \mathrm{dA}$ & $120,00 \mathrm{de} A$ & $106,25 \mathrm{~dB}$ & $94,25 \mathrm{dCD}$ & $105,54 d$ \\
\hline CNA 7800 & $80,75 \mathrm{eC}$ & $93,50 \mathrm{eB}$ & $109,75 \mathrm{eA}$ & $111,00 \mathrm{eA}$ & $107,25 \mathrm{dA}$ & $87,75 \mathrm{dBC}$ & $98,33 \mathrm{e}$ \\
\hline CNA 7801 & $91,25 \mathrm{deD}$ & 101,00deCD & $125,50 \mathrm{dA}$ & $117,25 \mathrm{de} A B$ & $110,75 \mathrm{cdBC}$ & $93,75 \mathrm{dD}$ & $106,58 \mathrm{~d}$ \\
\hline Caiapó & $120,25 \mathrm{aC}$ & $126,00 \mathrm{abBC}$ & $146,75 \mathrm{abA}$ & $148,25 \mathrm{abcA}$ & $135,00 \mathrm{aB}$ & $126,00 \mathrm{abBC}$ & $133,79 a$ \\
\hline R. Paranaíba & 113,00abD & $131,00 \mathrm{aC}$ & $146,50 \mathrm{abAB}$ & $150,50 \mathrm{abA}$ & $136,75 \mathrm{aBC}$ & $135,50 \mathrm{aC}$ & $135,54 \mathrm{a}$ \\
\hline Araguaia & $110,75 \mathrm{abD}$ & $126,50 \mathrm{abC}$ & $152,25 \mathrm{aA}$ & $153,50 \mathrm{aA}$ & $137,25 \mathrm{aB}$ & $135,25 \mathrm{aBC}$ & $135,92 \mathrm{a}$ \\
\hline \multirow[t]{2}{*}{ Média } & $100,83 \mathrm{D}$ & $114,06 \mathrm{C}$ & $133,97 \mathrm{~A}$ & $133,69 \mathrm{~A}$ & $124,03 \mathrm{~B}$ & $112,36 \mathrm{C}$ & 119,82 \\
\hline & & & & $1996 / 97$ & & & \\
\hline IAC 201 & $109,25 \mathrm{cdD}$ & $118,75 \mathrm{bcABC}$ & $124,41 \mathrm{bA}$ & $110,25 \mathrm{dCD}$ & $122,08 \mathrm{bcAB}$ & $113,41 \mathrm{aBCD}$ & $116,36 \mathrm{c}$ \\
\hline Carajás & $120,59 \mathrm{abB}$ & $125,17 \mathrm{bB}$ & $140,92 \mathrm{aA}$ & $123,42 \mathrm{bcB}$ & $121,33 \mathrm{cB}$ & $118,83 \mathrm{aB}$ & $125,04 \mathrm{~b}$ \\
\hline Guarani & $119,25 \mathrm{bcB}$ & $124,75 \mathrm{bB}$ & $140,50 \mathrm{aA}$ & $125,00 \mathrm{abB}$ & $125,25 \mathrm{bcB}$ & $117,00 \mathrm{aB}$ & $125,29 b$ \\
\hline IAC 202 & 98,99efCD & $115,25 \mathrm{bcdA}$ & $114,00 \mathrm{cA}$ & $113,58 \mathrm{cdAB}$ & $104,33 \mathrm{dBC}$ & $93,83 \mathrm{bD}$ & $106,67 d$ \\
\hline CNA 7800 & $89,16 \mathrm{fC}$ & $106,00 \mathrm{dA}$ & $105,67 \mathrm{cA}$ & $95,42 \mathrm{eBC}$ & $99,58 \mathrm{dAB}$ & $92,67 \mathrm{bBC}$ & $98,08 \mathrm{e}$ \\
\hline CNA 7801 & $99,41 \mathrm{deBC}$ & $112,25 \mathrm{cdA}$ & $114,67 \mathrm{bcA}$ & $108,08 \mathrm{dAB}$ & $106,75 \mathrm{dAB}$ & $97,33 \mathrm{dbC}$ & $106,42 d$ \\
\hline Caiapó & $127,08 \mathrm{abB}$ & $144,17 \mathrm{aA}$ & $143,75 \mathrm{aA}$ & $133,67 \mathrm{aB}$ & $131,50 \mathrm{abB}$ & $116,18 \mathrm{aC}$ & $132,72 \mathrm{a}$ \\
\hline R. Paranaíba & $129,25 \mathrm{abDE}$ & $150,92 \mathrm{aA}$ & $143,75 \mathrm{aAB}$ & $133,25 \mathrm{abCD}$ & $138,75 \mathrm{aBC}$ & $123,08 \mathrm{aE}$ & $136,50 \mathrm{a}$ \\
\hline Araguaia & $129,42 \mathrm{aBC}$ & $146,67 \mathrm{aA}$ & $146,08 \mathrm{aA}$ & $133,92 \mathrm{aB}$ & $138,41 \mathrm{aAB}$ & $120,58 \mathrm{aC}$ & $135,85 \mathrm{a}$ \\
\hline Média & $113,60 \mathrm{D}$ & $127,10 \mathrm{~B}$ & $130,42 \mathrm{~A}$ & $119,62 \mathrm{C}$ & $120,89 \mathrm{C}$ & $110,32 \mathrm{E}$ & 120,33 \\
\hline
\end{tabular}


elevadas na fase vegetativa das plantas, principalmente nos meses de dezembro e janeiro (Fig. 1), concordando, assim, com Terres \& Galli (1985) e Tanaka (1987).

Os resultados obtidos na avaliação do acamamento de plantas encontram-se nas Figs. 3 e 4, em que se pode observar que, de maneira geral, as semeaduras realizadas mais cedo - em setembro e outubro - ou mais tarde - fevereiro - apresentaram ausência ou baixo índice de acamamento na maioria das cultivares utilizadas. Estas épocas também apresentaram menores valores de altura das plantas (Tabela 2). As cultivares IAC 202 e CNA 7800 apresentaram ausência de acamamento em todas as épocas de semeadura nos dois anos de cultivo. Já a cultivar Guarani apresentou os maiores índices de acamamento, principalmente nas semeaduras realizadas nos meses de novembro, dezembro e janeiro. Arf (1993) também verificou que a cultivar Guarani apresentou acamamento total em todos os tratamentos utilizados, dificultando muito a operação da colheita, e sugerindo que a cultivar apresenta colmos fracos, considerando que outras cultivares com plantas mais altas apresentaram menores índices de acamamento. A cultivar CNA 7801 também apresentou ausência ou baixo índice de acamamento na maioria das épocas de semeadura, nos dois anos de cultivo.

Na Tabela 3 estão apresentados os dados obtidos quanto ao número de panículas por $\mathrm{m}^{2}$, em que se verifica efeito significativo da interação cultivar $\mathrm{x}$ época de semeadura somente no ano agrícola 1995/96, sendo que as cultivares Carajás e IAC 202 apresentaram os maiores números de panículas $/ \mathrm{m}^{2}$; porém não diferiram significativamente das cultivares Guarani, CNA 7800 e CNA 7801, e as semeaduras realizadas em novembro e dezembro propicia-

TABELA 3. Número de panículas por metro quadrado de nove cultivares de arroz em diferentes épocas de semeadura. Selvíria, MS, 1995/96 e 1996/97¹.

\begin{tabular}{|c|c|c|c|c|c|c|c|}
\hline \multirow[t]{2}{*}{ Cultivar } & \multicolumn{6}{|c|}{ Época de semeadura } & \multirow[t]{2}{*}{ Média } \\
\hline & Set. & Out. & Nov. & Dez. & Jan. & Fev. & \\
\hline \multicolumn{8}{|c|}{$1995 / 96$} \\
\hline IAC 201 & $175,25 \mathrm{AB}$ & $205,25 \mathrm{abcA}$ & $223,50 \mathrm{abA}$ & $204,50 \mathrm{abcA}$ & $141,00 \mathrm{bB}$ & $182,25 \mathrm{abAB}$ & $188,63 \mathrm{bcd}$ \\
\hline Carajás & $210,50 \mathrm{AB}$ & $221,50 \mathrm{abA}$ & $249,50 \mathrm{aA}$ & $232,00 \mathrm{abcA}$ & $165,25 \mathrm{abB}$ & $200,25 \mathrm{abAB}$ & $213,17 \mathrm{a}$ \\
\hline Guarani & $168,50 \mathrm{~B}$ & $201,50 \mathrm{abcAB}$ & $216,50 \mathrm{abAB}$ & $244,50 \mathrm{aA}$ & $200,25 \mathrm{aAB}$ & 201,00abAB & $205,38 \mathrm{ab}$ \\
\hline IAC 202 & $189,75 \mathrm{~A}$ & $229,75 \mathrm{abA}$ & $231,00 \mathrm{abA}$ & $237,25 \mathrm{abA}$ & $204,50 \mathrm{aA}$ & $219,75 \mathrm{aA}$ & $218,67 \mathrm{a}$ \\
\hline CNA 7800 & $156,50 \mathrm{C}$ & $196,50 \mathrm{abcBC}$ & $233,50 \mathrm{abAB}$ & $250,00 \mathrm{aA}$ & $178,50 \mathrm{abBC}$ & $189,75 \mathrm{abBC}$ & $200,79 \mathrm{abc}$ \\
\hline CNA 7801 & $184,00 \mathrm{~B}$ & $248,25 \mathrm{aA}$ & $195,75 \mathrm{abAB}$ & $237,25 \mathrm{abA}$ & $178,25 \mathrm{abB}$ & $211,25 \mathrm{abAB}$ & $209,13 \mathrm{ab}$ \\
\hline Caiapó & $167,75 \mathrm{AB}$ & $176,00 \mathrm{bcAB}$ & $211,75 \mathrm{abA}$ & $182,00 \mathrm{bcAB}$ & $157,75 \mathrm{abB}$ & $166,75 \mathrm{abAB}$ & $177,00 \mathrm{~d}$ \\
\hline R. Paranaíba & $167,50 \mathrm{AB}$ & $154,50 \mathrm{cB}$ & $209,00 \mathrm{abA}$ & $200,25 \mathrm{abc} A \mathrm{~B}$ & $167,00 \mathrm{ab} A B$ & $161,75 \mathrm{bAB}$ & $176,67 d$ \\
\hline Araguaia & $181,50 \mathrm{~A}$ & $174,00 \mathrm{bcA}$ & $186,00 \mathrm{bA}$ & $179,00 \mathrm{cA}$ & $179,50 \mathrm{abA}$ & $174,75 \mathrm{abA}$ & $179,13 \mathrm{~cd}$ \\
\hline Média & $177,92 \mathrm{D}$ & $200,86 \mathrm{BC}$ & $217,39 \mathrm{AB}$ & $218,53 \mathrm{~A}$ & $174,67 \mathrm{D}$ & $189,72 \mathrm{CD}$ & 196,50 \\
\hline \multicolumn{8}{|c|}{$1996 / 97$} \\
\hline IAC 201 & 193,12 & 294,37 & 206,25 & 151,88 & 173,12 & 177,50 & $182,71 \mathrm{abc}$ \\
\hline Carajás & 205,75 & 222,50 & 211,88 & 165,00 & 179,37 & 191,25 & $195,96 \mathrm{a}$ \\
\hline Guarani & 202,50 & 203,75 & 211,88 & 163,12 & 175,00 & 191,25 & $191,25 \mathrm{ab}$ \\
\hline IAC 202 & 156,87 & 203,12 & 154,38 & 158,12 & 171,25 & 178,12 & $170,31 \mathrm{bc}$ \\
\hline CNA 7800 & 182,50 & 190,62 & 173,75 & 161,87 & 192,50 & 176,88 & $179,69 \mathrm{abc}$ \\
\hline CNA 7801 & 203,75 & 206,82 & 196,88 & 166,88 & 207,50 & 193,75 & $195,94 a$ \\
\hline Caiapó & 155,00 & 208,75 & 178,12 & 162,50 & 166,25 & 195,63 & $177,71 \mathrm{abc}$ \\
\hline R. Paranaíba & 171,25 & 179,38 & 176,88 & 139,37 & 155,00 & 156,88 & $163,13 \mathrm{c}$ \\
\hline Araguaia & 181,87 & 169,38 & 183,12 & 155,00 & 153,12 & 183,12 & $170,94 \mathrm{bc}$ \\
\hline Média & $183,62 \mathrm{AB}$ & $197,64 \mathrm{~A}$ & $188,12 \mathrm{AB}$ & $158,19 \mathrm{C}$ & $174,79 \mathrm{~B}$ & $182,71 \mathrm{AB}$ & 180,85 \\
\hline
\end{tabular}


TABELA 4. Produção de grãos de nove cultivares de arroz em diferentes épocas de semeadura. Selvíria, MS, 1995/96 e 1996/971.

\begin{tabular}{|c|c|c|c|c|c|c|c|}
\hline \multirow[t]{2}{*}{ Cultivar } & \multicolumn{6}{|c|}{ Época de semeadura (1995/96) } & \multirow[t]{2}{*}{ Média } \\
\hline & Set. & Out. & Nov. & Dez. & Jan. & Fev. & \\
\hline \multicolumn{8}{|c|}{ 1995/96 } \\
\hline IAC 201 & $3.163 \mathrm{aCD}$ & $4.457 \mathrm{aA}$ & 4.195bcdAB & $3.019 \mathrm{cCD}$ & $2.240 \mathrm{bcD}$ & $3.321 \mathrm{abBC}$ & $3.399 \mathrm{bc}$ \\
\hline Carajás & $3.418 \mathrm{aB}$ & 3.790abB & $4.763 \mathrm{abcA}$ & 3.892abAB & $3.407 \mathrm{aB}$ & $3.383 \mathrm{abB}$ & $3.775 \mathrm{ab}$ \\
\hline Guarani & $2.563 \mathrm{abcB}$ & $3.566 \mathrm{abA}$ & $2.959 \mathrm{eAB}$ & $3.454 \mathrm{bAB}$ & $3.346 \mathrm{aAB}$ & $3.734 \mathrm{aA}$ & $3.270 \mathrm{~cd}$ \\
\hline IAC 202 & $2.130 \mathrm{bcC}$ & $3.132 \mathrm{bcB}$ & $4.319 \mathrm{bcdA}$ & $2.913 \mathrm{bcdBC}$ & $2.169 \mathrm{bcC}$ & 2.893abBC & $2.926 \mathrm{de}$ \\
\hline CNA 7800 & $2.580 \mathrm{abcC}$ & $3.102 \mathrm{bcBC}$ & $4.992 \mathrm{abA}$ & $3.767 \mathrm{bB}$ & 3.020abBC & $2.763 \mathrm{abC}$ & $3.370 \mathrm{bc}$ \\
\hline CNA 7801 & 2.779abcC & $4.503 \mathrm{aB}$ & $5.765 \mathrm{aA}$ & $4.832 \mathrm{aAB}$ & $2.903 \mathrm{abC}$ & $3.274 \mathrm{abC}$ & $4.009 \mathrm{a}$ \\
\hline Caiapó & 2.969abAB & $2.453 \mathrm{cdBC}$ & $3.801 \mathrm{cde} A$ & $2.263 \mathrm{cdBC}$ & $1.829 \mathrm{cC}$ & $2.449 \mathrm{bBC}$ & $2.627 \mathrm{ef}$ \\
\hline R. Paranaíba & $1.836 \mathrm{cC}$ & $2.274 \mathrm{cdBC}$ & $2.824 \mathrm{eAB}$ & $2.220 \mathrm{cdBC}$ & $2.026 \mathrm{bcBC}$ & $3.252 \mathrm{abA}$ & $2.405 f$ \\
\hline Araguaia & $2.122 \mathrm{cBC}$ & $2.025 \mathrm{dBC}$ & $3.305 \mathrm{deA}$ & $1.919 \mathrm{dBC}$ & $1.529 \mathrm{cC}$ & $2.467 \mathrm{bAB}$ & $2.228 \mathrm{f}$ \\
\hline Média & $2.618 \mathrm{C}$ & $3.256 \mathrm{~B}$ & $4.102 \mathrm{~A}$ & $3.142 B$ & $2.496 \mathrm{C}$ & $3.060 \mathrm{~B}$ & 3.112 \\
\hline \multicolumn{8}{|c|}{$1996 / 97$} \\
\hline IAC 201 & 4.509abcAB & $4.185 B$ & $5.571 \mathrm{abA}$ & $3.348 \mathrm{bB}$ & $4.152 \mathrm{bB}$ & 3.907abcB & $4.278 \mathrm{bcd}$ \\
\hline Carajás & $5.426 \mathrm{aAB}$ & $4.469 \mathrm{BC}$ & $5.753 \mathrm{abA}$ & $3.603 b C$ & 4.379abBC & $4.386 \mathrm{aBC}$ & $4.669 \mathrm{ab}$ \\
\hline Guarani & $5.494 \mathrm{aA}$ & $4.346 \mathrm{AB}$ & 4.822abcAB & 3.717abB & $3.953 \mathrm{bB}$ & 4.354abAB & $4.448 \mathrm{bc}$ \\
\hline IAC 202 & $4.026 \mathrm{bcdABC}$ & $4.233 \mathrm{AB}$ & $5.046 \mathrm{abcA}$ & $3.614 \mathrm{bBC}$ & 4.376abAB & $2.978 \mathrm{cdC}$ & $4.046 \mathrm{~cd}$ \\
\hline CNA 7800 & 4.469abcAB & $4.418 \mathrm{AB}$ & $5.279 \mathrm{abcA}$ & $3.391 \mathrm{bBC}$ & 4.557abAB & $2.880 \mathrm{cdC}$ & $4.165 \mathrm{bcd}$ \\
\hline CNA 7801 & 4.912abABC & 4.839BC & $6.037 \mathrm{aA}$ & $4.938 \mathrm{aABC}$ & $5.451 \mathrm{aAB}$ & $3.803 \mathrm{abcC}$ & $4.997 \mathrm{a}$ \\
\hline Caiapó & $3.257 \mathrm{cdB}$ & $4.973 \mathrm{~A}$ & $4.592 \mathrm{bcA}$ & $3.876 \mathrm{abAB}$ & $3.938 \mathrm{bAB}$ & $3.084 \mathrm{bcdB}$ & $3.953 \mathrm{cde}$ \\
\hline R. Paranaíba & $3.518 \mathrm{cdBC}$ & $4.451 \mathrm{AB}$ & $4.726 \mathrm{bcA}$ & $4.030 \mathrm{abAB}$ & $4.087 \mathrm{bAB}$ & $2.443 \mathrm{dC}$ & $3.876 \mathrm{de}$ \\
\hline Araguaia & $2.960 \mathrm{dBC}$ & $4.779 \mathrm{~A}$ & $4.092 \mathrm{cAB}$ & $3.176 \mathrm{bBC}$ & $3.680 \mathrm{bAB}$ & $2.116 \mathrm{dC}$ & $3.467 \mathrm{e}$ \\
\hline Média & $4.286 \mathrm{~B}$ & $4.521 \mathrm{~B}$ & $5.102 \mathrm{~A}$ & $3.744 \mathrm{C}$ & $4.285 \mathrm{~B}$ & $3.328 \mathrm{D}$ & 4.211 \\
\hline
\end{tabular}

ram a obtenção do maior número de panículas $/ \mathrm{m}^{2}$. No ano agrícola 1996/97, houve apenas efeito significativo quanto à cultivar e à época de semeadura, e os maiores números de panículas $/ \mathrm{m}^{2}$ foram obtidos pelas cultivares Carajás e CNA 7801, não diferindo significativamente das cultivares IAC 201, Guarani, CNA 7800 e Caiapó. Quanto às épocas de semeadura, o maior número de panículas $/ \mathrm{m}^{2}$ foi obtido com a semeadura realizada em outubro, que não diferiu estatisticamente das semeaduras realizadas em setembro, novembro e fevereiro. Os menores valores obtidos na semeadura realizada em dezembro pode ser explicado pela maior ocorrência de cupins, que causou redução na população de plantas, mesmo apesar da aplicação de inseticida granulado no sulco de semeadura. Na Tabela 3, observa-se, ainda, que a média geral do número de panículas $/ \mathrm{m}^{2}$ obtida em 1996/97 foi inferior à obtida no ano agrícola 1995/96.
Os resultados referentes à produção de grãos encontram-se na Tabela 4 , na qual se pode verificar que houve destaque para a cultivar CNA 7801 nos dois anos de cultivo, apresentando média geral de 4.009 e $4.997 \mathrm{~kg} / \mathrm{ha}$ nos anos agrícolas 1995/96 e 1996/97, respectivamente, não diferindo, portanto, estatisticamente, da cultivar Carajás, que apresentou $3.775 \mathrm{~kg} / \mathrm{ha}(1995 / 96)$ e $4.669 \mathrm{~kg} / \mathrm{ha}(1996 / 97)$. As cultivares IAC 201 e CNA 7800 também apresentaram destaque em produção, apresentando valores superiores a $3.300 \mathrm{~kg} / \mathrm{ha}(1995 / 96)$ e $4.100 \mathrm{~kg} / \mathrm{ha}(1996 / 97)$. No segundo ano de cultivo, a cultivar Guarani também se destacou das demais, apresentando produção de grãos de $4.448 \mathrm{~kg} / \mathrm{ha}$, e atingindo $5.494 \mathrm{~kg} / \mathrm{ha}$ na semeadura realizada em setembro/96. Na semeadura de novembro, esta cultivar apresentou a maioria das plantas acamadas por ocasião da colheita (50\% a $75 \%)$. No que se refere à 
época de semeadura, o comportamento foi semelhante nos dois anos de cultivo, com destaque para a semeadura no mês de novembro, em que a cultivar CNA 7801, que apresentou destaque em produção, atingiu 5.765 e $6.037 \mathrm{~kg} / \mathrm{ha}$ nos anos agrícolas de 1995/96 e 1996/97, respectivamente. Na semeadura realizada em novembro, as cultivares IAC 201, Carajás, IAC 202 e CNA 7800 também apresentaram destaque, com produção de grãos superior a 4.195 e 5.046 kg/ha em 1995/96 e 1996/97, respectivamente. Ainda na Tabela 5, pode-se observar que a média de produção de grãos, obtida em cada época de semeadura e geral, foi maior no ano agrícola 1996/97 em relação a 1995/96. A maior produção de grãos obtida em 1996/97 pode ser explicada pela melhor distribuição de chuvas ocorrida no período de cultivo (Fig. 2). Os dados obtidos são concordantes com Sant'Ana (1989) e Fornasieri Filho \& Fornasieri (1993).
O rendimento de inteiros está apresentado na Tabela 5 , onde verifica-se que houve destaque para a cultivar Caiapó nos dois anos de cultivo, pois apresentou média geral de 53,14\% (1995/96) e 63,36\% (1996/97). No primeiro ano de cultivo, houve destaque também para as cultivares IAC 201, IAC 202, Carajás e CNA 7801; e no segundo ano de cultivo, todas as cultivares apresentaram, como média geral, nas épocas de semeadura, valores superiores a $50 \%$ de rendimento de inteiros. Quanto às épocas de semeadura, os maiores valores de rendimento de inteiros foram obtidos na semeadura realizada em fevereiro, nos dois anos de cultivo. A média geral obtida nos dois anos de cultivo mostra que em 1996/97 o rendimento de inteiros obtido foi aproximadamente $10 \%$ maior que em 1995/96. Também neste caso a melhor distribuição de chuvas no ano agrícola 1996/97 pode explicar o maior rendimento de inteiros obtido.

TABELA 5. Rendimento de grãos inteiros de nove cultivares de arroz em diferentes épocas de semeadura. Selvíria, MS, 1995/96 e 1996/97¹.

\begin{tabular}{|c|c|c|c|c|c|c|c|}
\hline \multirow[t]{2}{*}{ Cultivar } & \multicolumn{6}{|c|}{ Época de semeadura } & \multirow[t]{2}{*}{ Média } \\
\hline & Set. & Out. & Nov. & Dez. & Jan. & Fev. & \\
\hline \multicolumn{8}{|c|}{$1995 / 96$} \\
\hline IAC 201 & $38,73 \mathrm{aC}$ & $50,53 \mathrm{aAB}$ & 42,49abBC & $43,26 \mathrm{aBC}$ & 49,55abABC & $59,63 \mathrm{~A}$ & $47,37 \mathrm{~b}$ \\
\hline Carajás & $32,89 \mathrm{abD}$ & $48,34 \mathrm{aBC}$ & $41,59 \mathrm{bCD}$ & $44,26 \mathrm{aBC}$ & $54,59 \mathrm{abAB}$ & $63,38 \mathrm{~A}$ & $47,51 b$ \\
\hline Guarani & $22,10 b C$ & $43,09 \mathrm{abB}$ & $28,14 \mathrm{cC}$ & $30,70 \mathrm{bC}$ & $48,26 \mathrm{bB}$ & $60,72 \mathrm{~A}$ & $38,83 d$ \\
\hline IAC 202 & $41,74 \mathrm{aB}$ & $44,43 \mathrm{aB}$ & $46,53 \mathrm{abB}$ & $51,55 \mathrm{aAB}$ & $49,64 \mathrm{abB}$ & $61,01 \mathrm{~A}$ & $49,15 \mathrm{ab}$ \\
\hline CNA 7800 & $33,37 \mathrm{abC}$ & $40,92 \mathrm{abBC}$ & $43,10 \mathrm{abBC}$ & $47,99 \mathrm{aB}$ & $50,23 \mathrm{abAB}$ & $60,16 \mathrm{~A}$ & $45,96 \mathrm{bc}$ \\
\hline CNA 7801 & $32,56 \mathrm{abC}$ & $48,12 \mathrm{aB}$ & 46,42abB & $48,99 \mathrm{aB}$ & 49,90abB & $61,53 \mathrm{~A}$ & $47,92 b$ \\
\hline Caiapó & $39,57 \mathrm{aD}$ & $46,61 \mathrm{aCD}$ & $54,37 \mathrm{aBC}$ & $50,27 \mathrm{aBCD}$ & $59,88 \mathrm{abAB}$ & $68,17 \mathrm{~A}$ & $53,14 \mathrm{a}$ \\
\hline R. Paranaíba & $26,28 \mathrm{bD}$ & $31,14 \mathrm{bcCD}$ & $39,51 \mathrm{bcBC}$ & $49,10 \mathrm{aB}$ & $60,79 \mathrm{aA}$ & $69,15 \mathrm{~A}$ & $45,99 \mathrm{bc}$ \\
\hline Araguaia & $24,57 \mathrm{bDE}$ & $22,97 \mathrm{cE}$ & $34,55 \mathrm{bcCD}$ & $45,46 \mathrm{aBC}$ & $55,01 \mathrm{abAB}$ & $65,73 \mathrm{~A}$ & $41,38 \mathrm{~cd}$ \\
\hline Médias & $32,42 \mathrm{E}$ & $41,79 \mathrm{D}$ & $41,85 \mathrm{D}$ & $45,73 \mathrm{C}$ & $53,09 \mathrm{~B}$ & $63,28 \mathrm{~A}$ & 46,36 \\
\hline \multicolumn{8}{|c|}{$1996 / 97$} \\
\hline IAC 201 & $50,99 \mathrm{cA}$ & $53,14 \mathrm{bcA}$ & 49,66bcdAB & $43,58 \mathrm{~dB}$ & $54,67 \mathrm{bcdA}$ & $49,53 \mathrm{cAB}$ & $50,26 f$ \\
\hline Carajás & $54,58 \mathrm{bcAB}$ & $59,66 \mathrm{abAB}$ & $45,38 \mathrm{cdC}$ & $55,25 \mathrm{bcAB}$ & $50,54 \mathrm{cdBC}$ & $59,72 \mathrm{abA}$ & 54,19 de \\
\hline Guarani & $51,14 \mathrm{cC}$ & $58,88 \mathrm{abAB}$ & $42,25 \mathrm{dD}$ & $52,78 \mathrm{cBC}$ & $46,96 \mathrm{dCD}$ & $61,13 \mathrm{abA}$ & $52,19 \mathrm{ef}$ \\
\hline IAC 202 & $60,50 \mathrm{ab}$ & $56,83 \mathrm{ab}$ & $54,28 \mathrm{ab}$ & $58,83 \mathrm{abc}$ & $61,40 \mathrm{ab}$ & $57,08 \mathrm{bc}$ & $58,15 \mathrm{bc}$ \\
\hline CNA 7800 & $56,53 \mathrm{bcA}$ & $45,52 \mathrm{cB}$ & $53,36 \mathrm{abA}$ & $58,88 \mathrm{abcA}$ & $53,16 \mathrm{cdA}$ & $58,81 \mathrm{abA}$ & $54,37 \mathrm{de}$ \\
\hline CNA 7801 & $57,68 \mathrm{bcA}$ & $48,19 \mathrm{cB}$ & $55,70 \mathrm{abA}$ & $60,08 \mathrm{abcA}$ & $56,81 \mathrm{abcA}$ & $58,00 \mathrm{abA}$ & $56,07 \mathrm{~cd}$ \\
\hline Caiapó & $65,85 \mathrm{a}$ & $62,83 \mathrm{a}$ & $58,71 \mathrm{a}$ & $63,57 \mathrm{a}$ & $63,83 \mathrm{a}$ & $65,40 \mathrm{a}$ & $63,36 \mathrm{a}$ \\
\hline R. Paranaíba & $60,71 \mathrm{abAB}$ & $62,16 \mathrm{aA}$ & $53,67 \mathrm{abB}$ & $62,80 \mathrm{abA}$ & $61,53 \mathrm{abA}$ & $57,07 \mathrm{bcAB}$ & $59,66 b$ \\
\hline Araguaia & $54,31 \mathrm{bcBC}$ & $59,93 \mathrm{abAB}$ & $51,23 \mathrm{abcC}$ & $59,86 \mathrm{abcAB}$ & $61,00 \mathrm{abAB}$ & $65,33 \mathrm{aA}$ & $58,61 b c$ \\
\hline Médias & $56,92 \mathrm{AB}$ & $56,35 \mathrm{~B}$ & $51,58 \mathrm{C}$ & $57,29 \mathrm{AB}$ & $56,66 \mathrm{~B}$ & $59,12 \mathrm{~A}$ & 56,32 \\
\hline
\end{tabular}




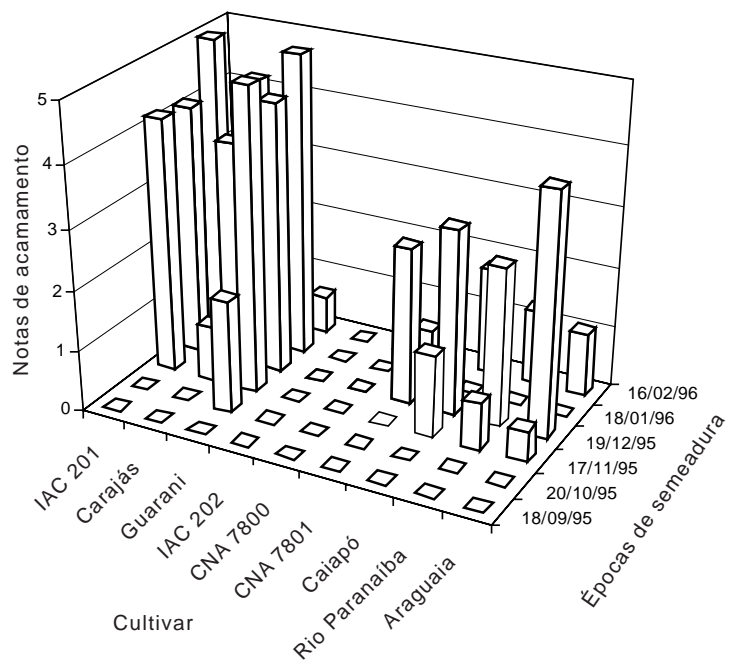

FIG. 3. Notas de acamamento de plantas de arroz de nove cultivares semeadas em diferentes épocas. Ano Agrícola 95/96.

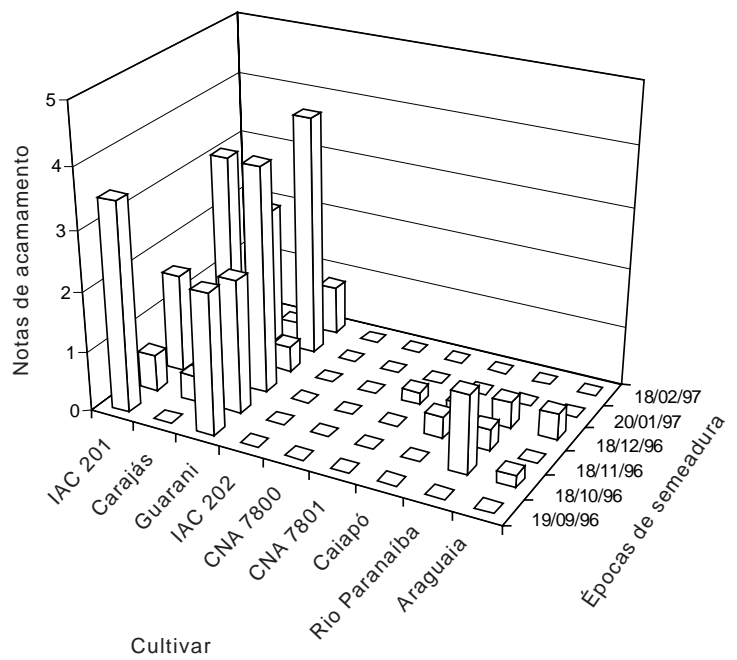

FIG. 4. Notas de acamamento de plantas de arroz de nove cultivares semeadas em diferentes épocas. Ano Agrícola 96/97.

\section{CONCLUSÕES}

1. As cultivares CNA 7801, Carajás, IAC 201, CNA 7800 e IAC 202 apresentam comportamento superior no cultivo de sequeiro irrigado por aspersão.
2. A semeadura realizada em novembro propicia a obtenção de produtividade mais elevada.

3. Semeaduras antecipadas (setembro-outubro) ou retardadas (fevereiro) causam menores índices de acamamento.

4. As cultivares IAC 202, CNA 7800 e CNA 7801 apresentam ausência ou baixo índice de acamamento, nas diferentes épocas de semeadura.

5. Semeadura retardada (fevereiro) oferece maior rendimento de inteiros.

\section{REFERÊNCIAS}

AMARAL, A.S. Semeadura do arroz. Pelotas : EmbrapaCPACT, 1979. 7p. (Embrapa-CPACT. Comunicado Técnico, 3).

ARF, O. Efeitos de densidade populacional e adubação nitrogenada sobre o comportamento de cultivares de arroz irrigado por aspersão. Ilha Solteira : UNESP-Faculdade de Engenharia de Ilha Solteira, 1993. 63p. Tese de Livre Docência.

CHANG, T.T.; OKA, H.I. Genetic variouness in the climatic adaptation of rice cultivar. In: INTERNATIONAL RICE RESEARCH INSTITUTE (Los Baños, Filipinas). Climate and rice. Los Baños, 1976. p.87109.

CHAUDHARY, T.N.; SODHI, J.S. Effect of weather factors on different dates in humid tropical area. Il Riso, Milan, v.28, p.255-263, 1979.

FARIA, R.T. Tensiômetro: construção, instalação e utilização: um aparelho simples para se determinar quando irrigar. Londrina : IAPAR, 1987. 23p. (IAPAR. Circular Técnica, 56).

FORNASIERI FILHO, D.; FORNASIERI, J.L. Manual da cultura do arroz. Jaboticabal : FUNEP, 1993. $221 \mathrm{p}$.

INFELD, J.A. Influência da temperatura e da radiação solar na produtividade do arroz irrigado. In: REUNIÃO DA CULTURA DO ARROZ IRRIGADO, 16., 1987, Balneário de Camboriú. Anais. Florianópolis : EMPASC, 1987. p.148-154.

INFELD, J.A. Produtividade de seis cultivares de arroz irrigado em função de épocas de semeadura. In: REUNIÃO DA CULTURA DO ARROZ IRRIGADO, 13., 1984, Balneário de Camboriú. Anais. Florianópolis : EMPASC, 1984. p.174-179. 
MORAIS, O.P.; SOUZA, I.F.; SILVEIRA, J.F. Época de plantio para a cultura do arroz (Oryza sativa L.) irrigado em Minas Gerais. In: EPAMIG (Belo Horizonte, MG). Projeto arroz: relatório anual 75/76. Belo Horizonte, 1978. p.65-74.

NAKAO, W.S. Manejo de água na cultura do arroz (Oryza sativa L.) irrigado por aspersão. Ilha Solteira : UNESP-Faculdade de Engenharia de Ilha Solteira,1995. 44p.

OLIVEIRA, G.S. Efeito de densidade de semeadura no desenvolvimento de cultivares de arroz (Oryza sativa $\mathbf{L}$.) em condições de sequeiro e irrigado por aspersão: análise comparativa de custos. Ilha Solteira : UNESP-Faculdade de Engenharia de Ilha Solteira, 1994. 45p.

PINHEIRO, B.S.; STEINMETZ, S.; STONE, L.F.; GUIMARÃES, E.P. Tipo de planta, regime hídrico e produtividade do arroz de sequeiro. Pesquisa Agropecuária Brasileira, Brasília, v.20, n.1, p.8795, jan. 1985.

RAIJ, B. van; QUAGGIO, J.A. Métodos de análise de solo para fins de fertilidade. Campinas : Instituto Agronômico, 1983. 31p.
SANT'ANA, E.P. Cultivo do arroz irrigado por aspersão. Informe Agropecuário, Belo Horizonte, v.14, p.71$75,1989$.

SANTOS, G.; BUENO, L.G.; UMBELINO, G.M. Estudo de três cultivares de arroz irrigado em oito épocas de semeadura. Goiânia : EMGOPA, 1978. 7p. (EMGOPA. Comunicado Técnico, 5).

TANAKA, R. Temperature and response in growth of young seedlings and mid-aged seedlings of the rice plants. Tohoku National Agricultural Experiment Station Bulletin, Marioka, v.76, p.57-88, 1987.

TERRES, A.L.; GALLI, J. Os efeitos do frio em cultivares de arroz irrigado no Rio Grande do Sul. In: EMBRAPA. Centro de Pesquisa Agropecuária de Terras Baixas e Clima Temperado (Pelotas, RS). Fundamentos para a cultura do arroz irrigado. Campinas : Fundação Cargill, 1985. p.83-94.

VERGARA, B.S. Physiological and morphological adaptability of rice varieties to climate. In: INTERNATIONAL RICE RESEARCH INSTITUTE (Los Baños, Filipinas). Climate and rice. Los Baños, 1976. p.67-83.

YOSHIDA, S. Climatic environment and its influence. In: INTERNATIONAL RICE RESEARCH INSTITUTE (Los Baños, Filipinas). Fundamentals of rice crop science. Los Baños, 1981. p.65-110. 